\title{
Identifying practices and strategies to increase HIV testing among Haitian migrant communities
}

\author{
Jonathan Santiago ${ }^{1,2^{*}}$, A Adrien ${ }^{1}$, C Spigner ${ }^{2}$, A Katz ${ }^{2}$ \\ From $16^{\text {th }}$ International Symposium on HIV and Emerging Infectious Diseases \\ Marseille, France. 24-26 March 2010
}

\section{Background}

Quebecers of Haitian origin (QHO) are disproportionately infected with HIV/AIDS as a result of socio-economic, cultural, and migratory factors. The Public Health Department of Montreal (PHDM) sought to identify practices and strategies to increase HIV testing in Haitian migrant communities.

\section{Methods}

Key informant interviews were conducted with representatives of community-based organizations providing HIV/AIDS services to the largest Haitian communities in the USA (New York, Boston, and Miami). A thematic analysis was completed to categorize qualitative data subsequently grouped into themes.

\section{Results}

- Rapid HIV testing had a significant role in decreasing the likelihood of Haitians not returning for their results due to stigma. It was also the preferred method of HIV testing for community level interventions due to its flexibility, mobility, and ease in carrying out.

- Group level interventions (GLI) targeting demographic groups were effective in increasing HIV/ AIDS education, promoting behavior change, and decreasing stigma in Haitian migrant communities. Based on social cognitive learning theory, GLIs use curricula that are non-judgmental, culturally sensitive, and linguistically appropriate.

- Community level interventions (CLI) in Haitian migrant communities engaged large audiences and increased HIV testing among populations who do not access conventional testing. CLIs included the use of mobile HIV testing vans, health conferences and fairs providing HIV testing, and outreach workers to conduct street outreach.

- Key informants advocated partnerships with clinics, hospitals, churches, and the business community. Many interventions to increase HIV/AIDS education and testing were conducted with stakeholders due to their unique capacity to mobilize and influence Haitian migrant communities.

- Collaborating with local Haitian media to design culturally competent public service announcements were seen as a valuable and cost-effective method to provide HIV/AIDS education, promote HIV testing, and recruit participants for GLIs and CLIs.

\section{Discussion}

Increasing HIV testing among QHO requires a comprehensive strategy designed and implemented by a multitude of stakeholders. Strategies to address stigma and low HIV/AIDS education are best tackled via GLIs and CLIs implemented by organizations trusted by QHO. The PHDM should also re-examine current HIV testing policy in Quebec and consider increasing access to rapid HIV testing.

\section{Author details}

${ }^{1}$ Agence de la santé et des services sociaux de Montréal, Montréal, Canada. ${ }^{2}$ University of Washington School of Public Health, Seattle, USA.

Published: 11 May 2010

doi:10.1186/1742-4690-7-S1-P111

Cite this article as: Santiago et al:. Identifying practices and strategies to increase HIV testing among Haitian migrant communities. Retrovirology 2010 7(Suppl 1):P111.

\footnotetext{
* Correspondence: jsanti25@gmail.com

${ }^{1}$ Agence de la santé et des services sociaux de Montréal, Montréal, Canada
} 